\title{
INTERPOLATION RESTRICTED TO DECREASING FUNCTIONS AND LORENTZ SPACES*
}

\author{
by JOAN CERDĀ and JOAQUIM MARTÍN
}

(Received 21st November 1996)

\begin{abstract}
For the real interpolation method, we identify the interpolated spaces of couples of classical Lorentz spaces through interpolation of the corresponding weighted $L_{p}$-spaces restricted to decreasing functions.
\end{abstract}

1991 Mathematics subject classification: 46M35, 46E30.

\section{Introduction}

Throughout this paper a decreasing function is a non-increasing and non-negative function on $\mathbf{R}^{+}=(0, \infty)$, which is endowed with Lebesgue measure, $L_{0}$ represents the vector lattice of (equivalence classes of) all measurable real functions on $\mathbf{R}^{+}$, and $L_{p}=L_{p}\left(\mathbf{R}^{+}\right)$.

We say that $X$ is a quasi-normed function space if it is a quasi-normed space and a linear subspace of $L_{0}$, such that, if $|f| \leq|g|, g \in X$ and $f \in L_{0}$, then $f \in X$ and $\|f\|_{X} \leq\|g\|_{X}$. In our examples, $X$ will have the Fatou property:

$$
\text { If } f_{n} \in X, 0 \leq f_{n} \uparrow f \text { a.e. and } \sup \left\|f_{n}\right\|_{X}<\infty \text {, then } f \in X \text { and }\left\|f_{n}\right\|_{X} \uparrow\|f\|_{X} .
$$

In this case, $X$ is complete, i.e., it is a quasi-Banach function space.

The cone of all decreasing functions of $X$ is denoted $X^{d}$. Here we are mainly interested with the case

$$
X=L_{p}(\omega)=\left\{f \in L_{0} ;\|f\|_{L_{p}(\omega)}=\|f \omega\|_{p}<\infty\right\} \quad(0<p \leq \infty),
$$

where $0<\omega \in L_{0}$, a weight on $\mathbf{R}^{+}$, and $\|\cdot\|_{p}$ is the usual quasi-norm on $L_{p}\left(\mathbf{R}^{+}\right)$.

Operators on these spaces restricted to decreasing functions have been used by several authors (cf. [2], [19], [7], etc.) to characterize when a variety of classical operators are bounded on Lorentz spaces associated to pairs $(u, \omega)$ of weights,

- This work has been partially supported by DGICYT, Grant PB94-0879 and by Suport a Grups de Recerca 1997 GR 00185. 


$$
\Lambda_{u}^{p}(\omega)=\left\{f \in L_{0} ;\|f\|_{\Lambda_{u}^{p}(\omega)}=\left\|f_{u}^{*}\right\|_{L_{p}(\omega)}<\infty\right\}
$$

where $f_{u}^{*}(x)=\inf \left\{\lambda>0 ; \int_{[|f|>\lambda\}} u(t) d t \leq x\right\}$. We write $f^{*}=f_{u}^{*}$ and $\Lambda^{p}(\omega)=\Lambda_{u}^{p}(\omega)$ if $u(t) \equiv 1$.

Conversely, the boundedness of operators $T$ acting on decreasing functions of $L_{p}(\omega)$ has been studied in [6] by considering the associate operator $\tilde{T}(f)=T\left(f_{u}^{*}\right)$ on $\Lambda_{u}^{p}(\omega)$.

On the other hand, interpolation of operators on weighted $L_{p}$-spaces restricted to monotone functions appear in [1], [11] and [21] in connection with some integral operators, and in [8, Theorem 2] we have seen how the boundedness of operators of Hardy type is enough to obtain interpolation results for cones of decreasing functions (cf. also Lemma 2 and Remark 3 below.) This type of interpolation was first used in [18] to present a unified account of some results about Fourier series with positive coefficients.

For a given pair $\bar{X}=\left(X_{0}, X_{1}\right)$ of quasi-normed function spaces, let $\bar{X}^{d}=\left(X_{0}^{d}, X_{1}^{d}\right)$, the corresponding pair of cones of decreasing functions, and $\Sigma\left(\bar{X}^{d}\right)=X_{0}^{d}+X_{1}^{d}$. For every $g \in \Sigma\left(\bar{X}^{d}\right)$, we denote

$$
K^{d}(g, t)=K\left(g, t ; \bar{X}^{d}\right)=\inf \left\{\left\|g_{0}\right\|_{0}+t\left\|g_{1}\right\|_{1} ; g=g_{0}+g_{1}, g_{j} \in X_{j}^{d}(j=0,1)\right\} .
$$

This $K^{d}$-functional is used to construct

$$
\left(\bar{X}^{d}\right)_{f, q}=\left\{g \in \Sigma\left(\bar{X}^{d}\right) ;\|g\|_{f, q, d}=\left\|\frac{K^{d}(g, t)}{f(t)}\right\|_{L_{q}\left(\frac{d}{t}\right)}<\infty\right\},
$$

if $0<q \leq \infty$ and $f$ a function parameter in the sense of [12]. We recall that this means that $f: \mathbf{R}^{+} \rightarrow \mathbf{R}^{+}$is continuous and $\bar{f}(t)=\sup f(t s) / f(s)<\infty$. As usual we write $\left(\bar{X}^{d}\right)_{9 . q}$ for $\left(\bar{X}^{d}\right)_{f, q}$ when $f(t)=t^{9}(0<\vartheta<1)$.

To deal with these classes $\left(\bar{X}^{d}\right)_{f, q}$, a useful tool is the following elementary decomposition lemma (cf. [8, Lemma 1]).

Lemma 1. Let $f, g, h \in L_{0}$ be decreasing functions such that $f \leq g+h$. Then $f=f_{0}+f_{1}$ with $f_{0}$ and $f_{1}$ decreasing, $f_{0} \leq g$ and $f_{1} \leq h$ (almost everywhere).

There is no general way to identify $\left(\bar{X}^{d}\right)_{f, q}$, even if the usual interpolation space $\bar{X}_{f, p}$ by the K-method is known, but for a number of important cases $\left(\bar{X}^{d}\right)_{f, q}=\left(\bar{X}_{f, q}\right)^{d}$.

Obviously this is true if $\bar{X}$ satisfies the following two conditions:

(a) $\Sigma(\bar{X})^{d} \subset \Sigma\left(\bar{X}^{d}\right)$, hence $\Sigma(\bar{X})^{d}=\Sigma\left(\bar{X}^{d}\right)$,

(b) $K^{d}(g, t) \leq C K(g, t)$, hence $K^{d}(g, t) \simeq K(g, t)$, when $g \in \Sigma\left(\bar{X}^{d}\right)$ and $t>0$,

for some constant $C=C(\bar{X})>0$. In this case $\bar{X}^{d}$ (or $\bar{X}$ as in [8]) is said to be a Marcinkiewicz pair.

We say that $T: \bar{X}^{d} \rightarrow \bar{X}$ is a bounded quasi-linear operator if it is an operator 
$T: \Sigma(\bar{X})^{d} \rightarrow \Sigma(\bar{X})$ such that $|T(f+g)| \leq C(|T f|+|T g|)$, and $T\left(X_{j}^{d}\right) \subset X_{j}$ with $\|T f\|_{X_{j}} \leq M_{j}\|f\|_{X_{j}}(j=0,1)$.

Lemma 2. If there exists a bounded quasi-linear operator $T: \bar{X}^{d} \rightarrow \bar{X}$ such that

(i) $g \in \Sigma(\bar{X})^{d}$ implies $g \leq T g$, and

(ii) $g \geq 0$ implies $\mathrm{Tg}$ decreasing,

then $\bar{X}^{d}$ is a Marcinkiewicz pair.

Proof. Let $g=f_{0}+f_{1} \in \Sigma(\bar{X})^{d}$, with $0 \leq f_{j} \in X_{j}(j=0,1)$. Then $g \leq C\left(T f_{0}+T f_{1}\right)$ with $T f_{j} \in X_{j}^{d}$ and from Lemma 1 we obtain $g=g_{0}+g_{1}$ with $g_{j} \in X_{j}^{d}$ such that $g_{j} \leq C T f_{j}$. Thus $g \in \Sigma\left(\bar{X}^{d}\right)$ and $K^{d}(g, t) \leq C K(g, t)$.

A typical choice for $T$ is $T=P+Q=P Q=Q P$, where $P$ and $Q$ are the Hardy operator and its adjoint

$$
P g(t)=\frac{1}{t} \int_{0}^{t} g(s) d s, \quad Q g(t)=\int_{t}^{\infty} g(s) \frac{d s}{s} .
$$

Remark 3. Let $D_{2} f(t)=f(t / 2)$. If $D_{2}$ and $Q$ are both bounded in $\bar{X}$, then $\bar{X}^{d}$ is a Marcinkiewicz pair, since $Q f$ is decreasing if $f \geq 0$ and, if $f$ is decreasing, $f(2 t) \leq$ $C \int_{t}^{2 s} f(s) \frac{d s}{s} \leq C \int_{t}^{\infty} f(s) \frac{d s}{s}$, whence $f \leq T f$ for $T=C D_{2} Q$ and Lemma 2 applies.

Remark 4. It is clear that a similar result to Lemma 1 holds for decreasing sequences instead of functions and, with the obvious changes, Lemma 2 is also true when $\bar{X}$ is a pair of quasi-normed lattices of sequences, such as weighted $\ell_{p}$ spaces.

Our aim is to study interpolation of classical Lorentz spaces $\left(\Lambda_{u}^{p_{0}}\left(\omega_{0}\right), \Lambda_{u}^{p_{1}}\left(\omega_{1}\right)\right)$ through interpolation of the corresponding cones $\left(L_{p_{0}}\left(\omega_{0}\right)^{d}, L_{p_{1}}\left(\omega_{1}\right)^{d}\right)$ of decreasing functions of weighted $L_{p}$-spaces. The paper is organized as follows:

Since Lorentz spaces are symmetric spaces associated to weighted $L_{p}$-spaces, in Section 2 we consider interpolation of symmetric spaces. Next, in Section 3 we prove that, under suitable conditions and for increasing weights, the couples $\left(L_{p_{0}}\left(\omega_{0}\right)^{d}, L_{p_{1}}\left(\omega_{1}\right)^{d}\right)$ are Marcinkiewicz pairs. Section 4 deals with decreasing weights. In Section 6 we identify the real interpolated spaces of classical Lorentz spaces as function spaces of the same type.

We refer to [4] and [5] for undefined notation and general facts about interpolation spaces, and to [3] and [14] for interpolation properties of Banach function spaces.

\section{Symmetric spaces}

The quasi-normed function space $X$ is said to be symmetric if and only if, whenever $g \in L_{0}$ and $f \in X$ are such that $g^{*} \leq f^{*}$, then $g \in X$ and $\|g\|_{X} \leq C\|f\|_{X}$, for some constant $C=C(X) \geq 1$. 
A symmetric space $X$ will be a quasi-Banach function space. All dilations $D_{\tau} f(t)=f(t / \tau)(\tau>0)$ are bounded in $X$, since they are bounded on F-spaces (cf. [13, Proposition 2]).

Theorem 5. If $X_{0}$ and $X_{1}$ are symmetric spaces, then $\Sigma(\bar{X})$, endowed with the usual sum quasi-norm, is also symmetric.

Proof. Let $f=f_{0}+f_{1} \in \Sigma(\bar{X})$ with $f_{j} \in X_{j}(j=0,1), g \in L_{0}, g^{*} \leq f^{*}$. Then $f^{*}(t) \leq f_{0}^{*}(t / 2)+$ $f_{1}^{*}(t / 2)$, and from Lemma 1 we obtain

$$
g^{*}=g_{0}+g_{1}, g_{j} \in X_{j}^{d}, g_{j} \leq D_{2}\left(f_{j}\right)^{*}=D_{2}\left(f_{j}^{*}\right) \quad(j=0,1) .
$$

Thus

$$
\left\|g^{*}\right\|_{\Sigma(\bar{X})} \leq C\left(\left\|g_{0}\right\|_{X_{0}}+\left\|g_{1}\right\|_{X_{1}}\right) \leq C\left\|D_{2}\right\|\left(\left\|f_{0}\right\|_{X_{0}}+\left\|f_{1}\right\|_{X_{1}}\right)
$$

with $\left\|D_{2}\right\|=\max \left(\left\|D_{2}\right\|_{X_{0}, X_{0}},\left\|D_{2}\right\|_{X_{1}, X_{1}}\right)$, and

$$
\left\|g^{*}\right\|_{\Sigma(\bar{X})} \leq C\left\|D_{2}\right\|\|f\|_{\Sigma(\bar{X})} .
$$

If $\lim _{\imath \uparrow \infty} g^{*}(t)=0$, there exists a measure-preserving transform $\sigma: \mathbf{R}^{+} \rightarrow \mathbf{R}^{+}$such that $g=g^{*} \circ \sigma$ (see [3, Corollary II.7.6]). Let $g^{*}=h_{0}+h_{1}, 0 \leq h_{j} \in X_{j}(j=0,1)$, such that

$$
\left\|h_{0}\right\|_{X_{0}}+\left\|h_{1}\right\|_{X_{1}} \leq\left\|g^{*}\right\|_{\Sigma(\bar{X})}+\varepsilon
$$

Since $g=h_{0} \circ \sigma+h_{1} \circ \sigma$ and $\left(h_{j} \circ \sigma\right)^{*}=h_{j}^{*}(j=0,1)$, from (3) and (4) we obtain

$$
\|g\|_{\Sigma(\bar{X})} \leq\left\|h_{0}^{*}\right\|_{X_{0}}+\left\|h_{1}^{*}\right\|_{X_{1}} \leq C\left(\left\|h_{0}\right\|_{X_{0}}+\left\|h_{1}\right\|_{X_{1}}\right) \leq C\left(\left\|D_{2}\right\|\|f\|_{\Sigma(\bar{X})}+\varepsilon\right)
$$

and it follows that $\|g\|_{\Sigma(\bar{X})} \leq C\left\|D_{2}\right\|\|f\|_{\Sigma(\bar{X})}$.

If $\lim _{t+\infty} g^{*}(t)>0$, then $\lim _{t+\infty} f^{*}(t)>0$ and $L_{\infty} \subset X_{0}+X_{1}$. For a given $\varepsilon>0$, we choose $|\beta(t)| \leq 1,|z(t)| \leq \varepsilon$ and a measure-preserving transform $\sigma: \mathbf{R}^{+} \rightarrow \mathbf{R}^{+}$such that $g=(f \circ \sigma) \beta+z($ see $[14$, Theorem II.2.1]). Then

$$
g=\left(f_{0} \circ \sigma\right) \beta+\left(f_{1} \circ \sigma\right) \beta+z \in X_{0}+X_{1}
$$

with $\|g\|_{\Sigma(\bar{X})} \leq C_{0}\left(\left\|f_{0}\right\|_{X_{0}}+\left\|f_{1}\right\|_{X_{1}}+\|z\|_{\Sigma(\bar{x})}\right)$, hence $\|g\|_{\Sigma(\bar{X})} \leq C\left(\|f\|_{\Sigma(\bar{X})}+\varepsilon\right)$.

Corollary 6. If $\bar{X}$ is a couple of symmetric spaces, $0<q \leq \infty$ and $f$ a function parameter, then $\bar{X}_{f, q}$ is also a symmetric space.

Remark 7. If $\bar{X}$ is a couple of symmetric spaces, it follows from (3) that $\bar{X}^{d}$ is a Marcinkiewicz pair (see also [8, Theorem 4]) since obviously $\Sigma(\bar{X})^{d} \subset \Sigma\left(\bar{X}^{d}\right)$ and, for any $g \in \Sigma(\bar{X})^{d}$, we can write $g=g_{0}+g_{1}$ with $0 \leq g_{j} \in X_{j}(j=0,1)$ and 


$$
\left\|g_{0}\right\|_{X_{0}}+\left\|g_{1}\right\|_{X_{1}} \leq\left\|D_{2}\right\|(K(g, t)+\varepsilon)
$$

thus $K^{d}(g, t) \leq C K(g, t)$.

The next theorem will be useful when applied to classical Lorentz spaces $\Lambda^{p}(\omega)$, since they appear when we "symmetrize" weighted $L^{p}$-spaces in the following way:

If $X$ is quasi-normed function space such that $D_{2}$ is bounded on $X$, then we define

$$
X^{s}=\left\{g \in L_{0} ; g^{*} \in X\right\} \text { with }\|g\|_{X^{s}}=\left\|g^{*}\right\|_{X} .
$$

Remark that $\|\cdot\|_{X^{s}}$ is a quasi-norm and, if $X$ has Fatou property, then $X^{s}$ has also this property, and it is complete.

Theorem 8. If $D_{2}$ is bounded on $X_{0}$ and $X_{1}$, and if $\bar{X}^{d}$ is a Marcinkiewicz pair, then

$$
\left(X_{0}^{s}, X_{1}^{s}\right)_{f, q}=\left(X_{0}, X_{1}\right)_{f, q}^{s},
$$

for any function parameter $f$ and any $0<q \leq \infty$.

Proof. From $X_{j}^{d}=X_{j}^{s d}$ and from Remark 6 we get

$$
\left(X_{0}, X_{1}\right)_{f, q}^{d}=\left(X_{0}^{d}, X_{1}^{d}\right)_{f, q}=\left(X_{0}^{s}, X_{1}^{s}\right)_{f, q}^{d}
$$

and $\left(X_{0}^{s}, X_{1}^{s}\right)_{f, q}=\left(X_{0}, X_{1}\right)_{f, q}^{s}$, since they are both symmetric with the same decreasing functions.

The boundedness of $D_{2}$ on $L_{p}(\omega)$ is equivalent to the $\Delta_{2}$-condition,

$$
\omega(2 t) \leq C \omega(t)
$$

As easy examples show (cf. [8, Examples 1 and 3] and Examples 9 and 15 below), some restrictions on the weights are needed for $\left(L_{p_{0}}\left(\omega_{0}\right)^{d}, L_{p_{1}}\left(\omega_{1}\right)^{d}\right)$ to be a Marcinkiewicz pair. It will be also convenient to consider weighted $L_{p}$-spaces for the measure $t^{-1} d t$ :

$$
L_{p}^{*}(\omega)=L_{p}\left(\omega(t) t^{1 / p}\right)=\left\{g \in L_{0} ; \int_{0}^{\infty}|g(t) \omega(t)|^{p} \frac{d t}{t}<\infty\right\}
$$

Example 9. For the increasing weights $\omega_{0}(s)=s$ and $\omega_{1}(s)=\max (1, s)$,

$$
L_{\mathrm{i}}^{*}\left(\omega_{0}\right)^{d}=L_{1}^{d}, L_{\mathrm{i}}^{*}\left(\omega_{1}\right)^{d}=L_{1}(\max (1 / s, 1))^{d}
$$

is not a Marcinkiewicz pair, since 


$$
\left(L_{1}^{*}\left(\omega_{0}\right), L_{1}^{*}\left(\omega_{1}\right)\right)_{9,1}^{d}=L_{1}\left(\max (1 / s, 1)^{9}\right)^{d} \neq\{0\}
$$

and $\left(L_{1}^{*}\left(\omega_{0}\right)^{d}, L_{1}^{*}\left(\omega_{1}\right)^{d}\right)_{9,1}=\{0\}$.

\section{Weighted $L_{p}$-spaces with increasing weights}

Lemma 10. If $\omega$ is an increasing weight and $0 \leq f \in L_{0}$, then

(1) $\sup _{s>0} f^{*}(s) \omega(s) \leq \sup _{s>0} f(s) \omega(s)$, and

(2) $\int_{0}^{\infty} f^{*}(s) \omega(s) d s \leq \int_{0}^{\infty} f(s) \omega(s) d s$.

Proof. For (2) we refer to [14, (2.39), p. 74].

For (1) assume that $f$ is a simple function,

$$
f=s_{n}=\sum_{i=1}^{n} a_{i} \chi_{\left(\alpha_{1}, \beta_{i}\right)} \quad\left(a_{i}>0, \beta_{i} \leq \alpha_{i+1}\right) .
$$

If $n=1$ the result is obvious. Let $n>1$ and

$$
s_{n}^{*}=s_{n-1}^{*}+a_{k} \chi_{\left[m, m+\beta_{k}-\alpha_{k}\right)}
$$

with $a_{k}=\min _{1 \leq i \leq n} a_{i}, m=\sum_{i \neq k}\left(\beta_{i}-\alpha_{i}\right), s_{n}=s_{n-1}+\alpha_{k} \chi_{\left[\alpha_{k}, \beta_{k}\right.}$.

Now all we need is to consider the following possible situations:

(a) $a_{k} \omega\left(\beta_{k}\right) \geq \sup s_{n-1} \omega$.

(b) $\sup s_{n} \omega=\sup s_{n-1} \omega$.

(c) $a_{k} \omega\left(m+\beta_{k}-\alpha_{k}\right) \geq \sup s_{n-1}^{*} \omega$.

(d) $\sup s_{n}^{*} \omega=\sup s_{n-1}^{*} \omega$.

E.g., if (a) and (b) hold true, in the case $\sup s_{n}^{*} \omega=\sup s_{n-1}^{*} \omega$, then by induction,

$$
\sup s_{n}^{*} \omega=\sup s_{n-1}^{*} \omega \leq \sup s_{n-1} \omega \leq \sup s_{n} \omega .
$$

In the other case

$$
\sup s_{n}^{*} \omega \leq a_{k} \omega\left(m+\beta_{k}-\alpha_{k}\right) \leq a_{n} \omega\left(m+\beta_{k}-\alpha_{k}\right) \leq a_{n} \omega\left(\beta_{n}\right) \leq \sup s_{n} \omega .
$$

Theorem 11. Let $0<p_{0}, p_{1} \leq \infty$. If $\omega_{0}, \omega_{1}$ are two increasing weights that satisfy the $\Delta_{2}$-condition (5), then $\left(L_{p_{0}}\left(\omega_{0}\right)^{d}, L_{p_{1}}\left(\omega_{1}\right)^{d}\right)$ is a Marcinkiewicz pair.

Proof. Let $f=f_{0}+f_{1} \in\left(L_{p_{0}}\left(\omega_{0}\right)+L_{p_{1}}\left(\omega_{1}\right)\right)^{d}$ with $f_{j} \in L_{p_{1}}\left(\omega_{j}\right)$. We apply Lemma 1 to $f \leq D_{2} f_{0}^{*}+D_{2} f_{1}^{*}$ and we obtain $f=g_{0}+g_{1}$ with $g_{j} \in L_{p_{j}}\left(\omega_{j}\right)^{d}$ such that $g_{j} \leq D_{2} f_{j}^{*}$. We apply Lemma 10 to get 


$$
\int_{0}^{\infty}\left(D_{2} f_{j}^{*}\right)^{p} \omega_{j} \leq C \int_{0}^{\infty}\left(f_{j}^{*}\right)^{p} \omega_{j} \leq C \int_{0}^{\infty}\left|f_{j}\right|^{p} \omega_{j} .
$$

Whence, $f \in L_{p_{0}}\left(\omega_{0}\right)^{d}+L_{p_{1}}\left(\omega_{1}\right)^{d}$ and

$$
K^{d}(f, t) \leq\left\|g_{0}\right\|_{L_{p_{0}}\left(\omega_{0}\right)}+t\left\|g_{1}\right\|_{L_{p_{1}}\left(\omega_{1}\right)} \leq C\left(\left\|f_{0}\right\|_{L_{p_{0}\left(\omega_{0}\right)}}+t\left\|f_{1}\right\|_{L_{p_{1}}\left(\omega_{1}\right)}\right) .
$$

Thus $K^{d}(f, t) \leq C K(f, t)$.

Remark 12. With the obvious changes in Lemma 10 and Theorem 11 we obtain the corresponding results for sequence spaces $\ell_{p_{0}}\left(\omega_{0}\right)$ and $\ell_{p_{1}}\left(\omega_{1}\right)$ with $\omega_{j}=\left\{\omega_{j}^{n}\right\}_{n=1}^{\infty}$ increasing weights which satisfy the $\Delta_{\mathbf{2}}$-condition.

Theorem 13. Let $0<p_{0}, p_{1} \leq \infty$. If $\omega_{0}, \omega_{1}$ are two increasing weights that satisfy the $\Delta_{2}$-condition (5) and if there exists $a>1$ such that

$$
\inf _{x>0} \frac{\omega_{j}(a x)}{\omega_{j}(x)}=\frac{1}{r}>1
$$

then $\left(L_{p_{0}}^{*}\left(\omega_{0}\right)^{d}, L_{p_{1}}^{*}\left(\omega_{1}\right)^{d}\right)$ is a Marcinkiewicz pair.

Proof. Assume $p_{0}, p_{1}<\infty$. For any $f \in L_{p_{1}}^{*}\left(\omega_{j}\right)^{d}$ we write

$$
\int_{0}^{\infty}\left|f(x) \omega_{j}(x)\right|^{p_{j}} \frac{d x}{x}=\sum_{n \in \mathbf{Z}} \int_{d^{n}}^{a^{n+1}}\left|f(x) \omega_{j}(x)\right|^{p_{1}} \frac{d x}{x}
$$

to obtain from the hypotheses

$$
\int_{0}^{\infty}\left|f(x) \omega_{j}(x)\right|^{p_{1}} \frac{d x}{x} \simeq \sum_{n \in \mathbf{Z}}\left|f\left(a^{n}\right) \omega_{j}\left(a^{n}\right)\right|^{p_{1}}
$$

and it follows that

$$
K\left(f, t ; L_{p_{0}}^{*}\left(\omega_{0}\right)^{d}, L_{p_{1}}^{*}\left(\omega_{1}\right)^{d}\right) \simeq K\left(\left(f\left(a^{n}\right)\right\}, t ; \ell_{p_{0}}\left(\omega_{0}\left(a^{n}\right)\right)^{d}, \ell_{p_{1}}\left(\omega_{1}\left(a^{n}\right)\right)^{d}\right) .
$$

Now, to apply the version of Lemma 2 for sequences (Remark 4) to prove that $\left(\ell_{p_{0}}\left(\omega_{0}\left(a^{n}\right)\right)^{d}, \ell_{p_{1}}\left(\omega_{1}\left(a^{n}\right)\right)^{d}\right)$ is a Marcinkiewicz pair, we define $\left(\tau_{n} \alpha\right)(k)=\alpha(k-n)$ and

$$
T(\alpha)=\left\{\sum_{k \geq n}\left|\alpha_{k}\right|\right\}_{n \in \mathbf{Z}}=\left\{\sum_{m \geq 0}\left|\tau_{-m} \alpha(n)\right|\right\}_{n \in \mathbb{Z}} .
$$

Then 


$$
\|T(\alpha)\|_{\left.\ell_{\eta}\left((\omega, \omega)\left(\alpha^{n}\right)\right)\right)} \leq\left(\sum_{m \geq 0}\left\|\tau_{-m}\right\| .\right)\|\alpha\|_{\ell_{m}\left(\left(\omega \omega,\left(\alpha^{n}\right)\right)\right)},
$$

with $\left\|\tau_{-m}\right\| \leq r^{-m}$, since $\omega_{j}\left(a^{k-n}\right) \leq r^{-n} w_{j}\left(a^{k}\right)$ and

$$
\left\|\tau_{-n} \alpha\right\|_{\ell_{\eta}\left(\left(\omega_{j}\left(a^{n}\right)\right\}\right)}^{p_{j}} \leq \sum_{k \in \mathbf{Z}}\left|\alpha(k) \omega_{j}\left(a^{k}\right)\right|^{p_{j}} r^{-n p_{j}}
$$

Thus we have a bounded sublinear operator $T$ on $\ell_{p_{j}}\left(\left\{\omega_{j}\left(a^{n}\right)\right\}\right)(j=0,1)$ such that $T(\alpha)$ is always decreasing and $\left|\alpha_{n}\right| \leq T(\alpha)_{n}$, and $\left(\ell_{p_{0}}\left(\omega_{0}\left(a^{n}\right)\right)^{d}, \ell_{p_{1}}\left(\omega_{1}\left(a^{n}\right)\right)^{d}\right)$ is a Marcinkiewicz pair.

Now, as in [5, Example 2.3.22], we obtain a bounded linear operator

$$
R: L_{p j}^{*}\left(\omega_{j}\right) \rightarrow \ell_{p}\left(\left\{\lambda_{j}^{n}\right\}\right) \quad(j=0,1)
$$

if $R f=\left\{(\log a)^{-1} \int_{a^{n}}^{a^{n+1}} f(s) \frac{d s}{s}\right\}_{n \in Z}$ and $\lambda_{j}^{n}=\left(\int_{a^{n}}^{a^{n+1}} \omega_{j}(s)^{p_{1}} \frac{d s}{s}\right)^{1 / p_{j}}$, since the weights $\omega_{j}$ are increasing and satisfy the $\Delta_{2}$-condition. Remark that $\lambda_{j}^{n} \simeq \omega_{j}\left(a^{n}\right)$ and $\ell_{p}\left(\left\{\lambda_{j}^{n}\right\}\right)=$ $\ell_{p_{j}}\left(\left\{\omega_{j}\left(a^{n}\right)\right\}\right)$.

If $f$ is decreasing, $f\left(a^{n+1}\right) \leq(\log a)^{-1} \int_{a^{n}}^{a^{n+1}} f(s) \frac{d s}{s} \leq f\left(a^{n}\right)$ and then

$$
K\left(R f, t ; \ell_{p_{0}}\left(\omega_{0}\left(a^{n}\right)\right), \ell_{p_{1}}\left(\omega_{1}\left(a^{n}\right)\right)\right) \leq C K\left(f, t ; L_{p_{0}}^{*}\left(\omega_{0}\right), L_{p_{1}}^{*}\left(\omega_{1}\right)\right),
$$

with $\tau_{-1}(\{f(n)\}) \leq R f$ and $\tau_{-1}$ bounded on $\ell_{p}\left(\omega_{j}\left(a^{n}\right)\right)$. Hence

$$
K\left(\{f(n)\}, t ; \ell_{p_{0}}\left(\omega_{0}\left(a^{n}\right)\right), \ell_{p_{1}}\left(\omega_{1}\left(a^{n}\right)\right)\right) \leq C K\left(f, t ; L_{p_{0}}^{*}\left(\omega_{0}\right), L_{p_{1}}^{*}\left(\omega_{1}\right)\right) .
$$

To prove the theorem in the case $\left(L_{p_{0}}^{*}\left(\omega_{0}\right)^{d}, L_{\infty}\left(\omega_{1}\right)^{d}\right)\left(0<p_{0}<\infty\right)$, consider the operator $P: L_{\infty}\left(\omega_{1}\right) \rightarrow \ell_{\infty}\left(\omega_{1}\left(a^{n}\right)\right)$ such that

$$
P f=\left\{\sup _{a^{n} \leq s<a^{n+1}}\left|f\left(a^{n}\right)\right|\right\}_{n \in \mathbf{Z}} .
$$

Remark 14. With a similar proof, $\left(L_{p_{0}}^{*}\left(\omega_{0}\right)^{d}, L_{p_{1}}^{*}\left(\omega_{1}\right)^{d}\right)$ is still a Marcinkiewicz pair if $\omega_{0}$ and $\omega_{1}$ are two weights which satisfy the $\Delta_{2}$-condition and with properties

$$
\inf _{x>0} \frac{\omega_{j}(a x)}{\omega_{j}(x)}>1, \sup _{n \in Z} \sup _{s, t \in\left[a^{+}, a^{+1}\right]} \frac{\omega_{j}(s)}{\omega_{j}(t)}<\infty,
$$

without any monotonicity condition on the weights.

\section{Weighted $L_{p}$-spaces with decreasing weights}

For decreasing weights it is easy to produce examples of couples of weighted $L_{p}$-spaces whose cones of decreasing functions are not Marcinkiewicz pairs. 
Example 15. Let $0<\theta<1$ and $\omega$ any integrable decreasing weight such that $\omega^{9} \in L_{1}$ (e.g., $\left.\omega(t)=e^{-t}\right)$. Then

$$
\left(L_{1}^{d}, L_{1}(\omega)^{d}\right)_{9,1} \neq\left(L_{1}, L_{1}(\omega)\right)_{9,1}^{d},
$$

since in this case $K^{d}(1, t)=t\|1\|_{L_{1}(\omega)}$ and $1 \notin\left(L_{1}^{d}, L_{1}(\omega)^{d}\right)_{9,1}$, but $1 \in L_{1}\left(\omega^{s}\right)^{d}=$ $\left(L_{1}, L_{1}(\omega)\right)_{9,1}^{d}$.

Remark 16. For increasing weights we have used a condition on the growth, the $\Delta_{2}$-condition. For a decreasing weight we shall consider the condition

$$
\frac{1}{s} \int_{0}^{s} \omega(t) d t \simeq \omega(s) \quad\left(\text { i.e., } \frac{1}{s} \int_{0}^{s} \omega(t) d t \leq C \omega(s)\right)
$$

which is a decrease property, since, as shown in [9], it is equivalent to

$$
\inf _{x>0} \frac{\omega(r x)}{\omega(x)}>\frac{1}{r} \text { for some constant } r>1 .
$$

Theorem 17. Let $0<p_{0}, p_{1}<\infty$ and let $\omega_{0}, \omega_{1}$ be two decreasing weights such that $\omega_{0}^{p_{0}}$ and $\omega_{1}^{p_{1}}$ satisfy the decrease condition (7). Then $\left(L_{p_{0}}\left(\omega_{0}\right)^{d}, L_{p_{1}}\left(\omega_{1}\right)^{d}\right)$ is a Marcinkiewicz pair.

Proof. For $\Phi_{j}(x)=\left(\int_{0}^{x} \omega_{j}^{p_{j}}(s) d s\right)^{1 / p_{j}}$ we have $L_{p_{1}}\left(\omega_{j}\right)=L_{p_{j}}^{*}\left(\Phi_{j}\right)$ with equivalent norms and we know from Theorem 13 that $\left(L_{p_{0}}^{*}\left(\Phi_{0}\right)^{d}, L_{p_{1}}^{*}\left(\Phi_{j}\right)^{d}\right)$ is a Marcinkiewicz pair.

Remark 18. In the case $p_{j} \geq 1$, in Theorem 17 we are under the conditions of Remark 3, since, as shown in [9], for any decreasing weight $\omega$ and $p \geq 1$, the following properties are equivalent:

(a) $Q: L_{p}(\omega)^{d} \rightarrow L_{p}(\omega)$, bounded.

(b) $Q: L_{p}(\omega) \rightarrow L_{p}(\omega)$, bounded.

(c) $\omega^{p}$ satisfies condition (7).

\section{Interpolation of Lorentz spaces}

In general it is not true that the interpolated space by the real K-method of a couple $\left(\Lambda^{p_{0}}\left(\omega_{0}\right), \Lambda^{p_{1}}\left(\omega_{1}\right)\right)$ of Lorentz spaces is the Lorentz space associated to the interpolated space of corresponding $L_{p}$-spaces. In Example 15 of a couple $\left(L_{1}\left(\omega_{0}\right)^{d}, L_{1}\left(\omega_{1}\right)^{d}\right)$ which is not a Marcinkiewicz pair,

$$
\left(\Lambda^{1}\left(\omega_{0}\right), \Lambda^{1}\left(\omega_{1}\right)\right)_{9.1} \neq \Lambda^{\prime}\left(\omega_{0}^{1-8} \omega_{1}^{8}\right)
$$


The results of the previous sections allow to give sufficient conditions to identify the interpolated spaces of couples of Lorentz spaces $\Lambda_{u}^{p}$ defined as in (1).

Theorem 19. Let $\left(\omega_{0}, \omega_{1}\right)$ be a couple of $\Delta_{2}$-weights and $0<p_{0}, p_{1} \leq \infty$, and assume that $\left(L_{p_{0}}\left(\omega_{0}\right)^{d},\left(L_{p_{1}}\left(\omega_{1}\right)^{d}\right)\right)$ is a Marcinkiewicz pair. Then, for any weight $u$,

$$
\left(\Lambda_{u}^{p_{0}}\left(\omega_{0}\right), \Lambda_{u}^{p_{1}}\left(\omega_{1}\right)\right)_{9, q}=\Lambda_{u}^{q}\left(\omega_{0}^{1-9} \omega_{1}^{q}\right)
$$

if $1 / q=(1-\vartheta) / p_{0}+\vartheta / p_{1}$ and $0<\vartheta<1$.

\section{Proof. Denote}

$$
K(f, t)=K\left(f, t ; \Lambda_{u}^{p_{0}}\left(\omega_{0}\right), \Lambda_{u}^{p_{1}}\left(\omega_{1}\right)\right)
$$

and

$$
K\left(f_{u}^{*}, t\right)=K\left(f_{L}^{*}, t ; L_{p_{0}}\left(\omega_{0}\right), L_{p_{1}}\left(\omega_{1}\right)\right)
$$

We only need to prove that $K(f, t) \simeq K\left(f_{u}^{*}, t\right)$ (cf. [4, Theorem 5.5.1]).

Obviously, $K\left(f_{u}^{*}, t\right) \leq c K(f, t)$ for any $f \in \Lambda_{u}^{p_{0}}\left(\omega_{0}\right)+\Lambda_{u}^{p_{1}}\left(\omega_{1}\right)$, since we can consider $f=f_{0}+f_{1}$ with

$$
\left\|f_{0}\right\|_{\Lambda_{u}^{p_{0}}\left(\omega_{0}\right)}+t\left\|f_{1}\right\|_{\Lambda_{u}^{P_{1}^{\prime}\left(\omega_{1}\right)}} \leq 2 K(f, t)
$$

and then $f_{u}^{*} \leq D_{2} f_{0 u}^{*}+D_{2} f_{1 u}^{*}$.

For the converse we consider two cases. First assume that $f_{u}^{*} \in L_{p_{0}}\left(\omega_{0}\right)+L_{p_{1}}\left(\omega_{1}\right)$ is such that $\lim _{x \rightarrow \infty} f_{u}^{*}(x)=0$.

Since $\left(L_{p_{0}}\left(\omega_{0}\right)^{d}, L_{p_{1}}\left(\omega_{1}\right)^{d}\right)$ is a Marcinkiewicz pair, we can consider a decomposition $f_{u}^{*}=f_{0}+f_{1}$ with $f_{j} \in L_{p}\left(\omega_{j}\right)^{d}$ with

$$
\left\|f_{0}\right\|_{L_{\phi_{0}}\left(\omega_{0}\right)}+t\left\|f_{1}\right\|_{L_{p_{1}}\left(\omega_{1}\right)} \leq 2 K^{d}\left(f_{u}^{*}, t\right) .
$$

In our case there exists a measure-preserving transformation $\sigma$ (cf. [3, Corollary II.7.6]) such that

$$
f=f_{u}^{*} \circ \sigma=f_{0} \circ \sigma+f_{1} \circ \sigma
$$

with $f_{j} \circ \sigma$ and $f_{j}$ equimeasurable. So $\left(f_{j} \circ \sigma\right)_{u}^{*}=f_{j}^{*}=f_{j}$ (a decreasing function), $\left\|f_{j} \circ \sigma\right\|_{\Lambda_{u}^{p_{j}}\left(\omega_{j}\right)}=\left\|f_{j}\right\|_{L_{p_{j}}\left(\omega_{j}\right)}$ and

$$
K(f, t) \leq\left\|f_{0} \circ \sigma\right\|_{\Lambda_{*}^{m_{0}}\left(\omega_{0}\right)}+t\left\|f_{1} \circ \sigma\right\|_{L_{*}^{\prime \prime}\left(\omega_{1}\right)} \leq\left\|f_{0}\right\|_{L_{p_{0}}\left(\omega_{0}\right)}+t\left\|f_{1}\right\|_{L_{p_{1}}\left(\omega_{1}\right)} \leq 2 K^{d}\left(f_{u}^{*}, t\right) .
$$

Assume now that $f_{u}^{*} \in L_{p_{0}}\left(\omega_{0}\right)+L_{p_{1}}\left(\omega_{1}\right)$ is such that $\lim _{x \rightarrow \infty} f_{u}^{*}(x)=f_{u}^{*}(\infty)>0$ (thus $L_{p_{0}}\left(\omega_{0}\right)+L_{p_{1}}\left(\omega_{1}\right)$ contains $\left.L_{\infty}\right)$ and let $f_{u}^{*}=f_{0}+f_{1}$ as above. In this case we consider 
any measure-preserving transformation $\sigma$ between $\mathbf{R}^{+}$with the measure $u$ and $\mathbf{R}^{+}$with Lebesgue measure with $\operatorname{supp} \sigma=\mathbf{R}^{+}$, and define $h=f_{u}^{*} \circ \sigma=f_{0} \circ \sigma+f_{1} \circ \sigma$. Again we obtain

$$
K(h, t) \leq 2 K^{d}\left(f_{u}^{*}, t\right)
$$

Since $h_{u}^{*}=f_{u}^{*}$,

$$
K(h, t) \simeq K\left(f_{u}^{*}, t\right)=K\left(h_{u}^{*}, t\right)
$$

and we observe that $K(f, t) \leq C K(h, t)$ by considering a measure-preserving transformation $\omega$ such that $|f(t)| \leq|h \circ \omega(t)|+\varepsilon$ (cf. [14, Theorem II.2.1]), hence

$$
K(f, t) \leq K(h \circ \omega, t)+\varepsilon K(1, t) \leq K(h, t)+\varepsilon \min \left(\|1\|_{L^{m^{\infty}\left(\omega_{0}\right)}}, t\|1\|_{L^{n_{1}}\left(\omega_{1}\right)}\right),
$$

for every $\varepsilon>0$.

Since $f_{u}^{*}(\infty) \in L_{p_{0}}\left(\omega_{0}\right)^{d}+L_{p_{1}}\left(\omega_{1}\right)^{d}$ and a decomposition of a constant function into sum of two decreasing functions necessarily gives constant terms, and $\left(L_{p_{0}}\left(\omega_{0}\right)^{d}, L_{p_{1}}\left(\omega_{1}\right)^{d}\right)$ is a Marcinkiewicz pair, one of the integrals $\|1\|_{L^{\infty_{0}}\left(\omega_{0}\right)},\|1\|_{L^{\prime 1}\left(\omega_{1}\right)}$ is finite.

Hence $\|1\|_{L^{\infty}\left(\omega_{0}\right)}<\infty$ or $\|1\|_{L^{\prime \prime}\left(\omega_{1}\right)}<\infty$ respectively.

Corollary 20. Let $\omega$ be any $\Delta_{2}$-weight and $0<p_{0}, p_{1} \leq \infty$. Then

$$
\left(\Lambda_{u}^{p_{0}}(\omega), \Lambda_{u}^{p_{1}}(\omega)\right)_{9, q}=\Lambda_{u}^{q}(\omega)
$$

if $1 / q=(1-\vartheta) / p_{0}+\vartheta / p_{1}$, for any weight $u$.

Proof. In [8, Remark 3] we show that, for any quasi-Banach function lattice $X$, $\left(X^{d}, L_{\infty}^{d}\right)$ is a Marcinkiewicz pair. Thus, so is $\left(L_{r}(\omega)^{d}, L_{\infty}^{d}\right)$ and, if we consider $0<r<p_{j}$ $(j=0,1)$, by reiteration (cf. [8, Corollary 2]) it follows that $\left(L_{p_{0}}(\omega)^{d}, L_{p_{1}}(\omega)^{d}\right)$ is also a Marcinkiewicz pair, and we can apply Theorem 19.

Observe that Theorem 19 holds also for Lorentz spaces on $\mathbf{R}^{n}$.

Moreover, as an application of Theorem 8 to the case of weighted $L_{p}$ spaces, since the interpolation of weighted $L_{p}$ spaces is well known (cf. [10]), we can state another description of the interpolated spaces of couples of Lorentz spaces. If $\omega, u$ and $v$ are three weights and $0<p \leq \infty$, define

$$
\Lambda_{u, v}^{p}(\omega)=\left\{f \in L_{0} ;\left(f^{*} v\right)_{u}^{*} \in L_{p}(\omega)\right\}
$$

with $\|f\|=\left\|\left(f^{*} v\right)_{u}^{*}\right\|_{L_{p}(\omega)}$. In the case $u=v=1, \Lambda_{u, v}^{p}(\omega)=\Lambda^{p}(\omega)$ and, when $v$ is decreasing, if $U(t)=\int_{0}^{s} u(s) d s, \Lambda_{u, v}^{p}(\omega)=\Lambda_{u}^{p}(\omega)^{s}=\Lambda^{p}\left(v \omega(U) u^{1 / p}\right)$, which is space $\Lambda^{p}\left(\omega^{\prime}\right)$ for a suitable weight $w^{\prime}$. Then we have: 
Remark 21. Let $\left(\omega_{0}, \omega_{1}\right)$ be a couple of $\Delta_{2}$-weights, $f$ a function parameter, $0<q<\infty$ and $0<p_{0}, p_{1} \leq \infty$, and assume that $\left(L_{p_{0}}\left(\omega_{0}\right)^{d}, L_{p_{1}}\left(\omega_{1}\right)^{d}\right)$ is a Marcinkiewicz pair. In this case, the interpolation results for weighted $L^{p}$ spaces can be used (see [15] and [16]) and we obtain:

(a) If $p_{0} \neq p_{1}$, then

$$
\left(\Lambda^{p_{0}}\left(\omega_{0}\right), \Lambda^{p_{1}}\left(\omega_{1}\right)\right)_{S, q}=\Lambda_{u, v}^{p}(\omega)
$$

where $v=\left(\omega_{0} / \omega_{1}\right)^{p_{0} p_{1} /\left(p_{1}-p_{0}\right)}, u=\left(\omega_{1}^{p_{1}} / \omega_{0}^{p_{0}}\right)^{1 /\left(p_{1}-p_{0}\right)}$ and $\omega=t^{1 / p_{0}} f\left(t^{1 / p_{0}-1 / p_{1}}\right)$.

If $1 / q=(1-\vartheta) / p_{0}+\vartheta / p_{1}(0<\vartheta<1)$, again

$$
\left(\Lambda^{p_{0}}\left(\omega_{0}\right), \Lambda^{p_{1}}\left(\omega_{1}\right)\right)_{\vartheta, q}=\Lambda^{q}\left(\omega_{0}^{1-\vartheta} \omega_{1}^{9}\right)
$$

(b) If $p_{0}=p_{1}=p$, then

$$
\left(\Lambda^{p}\left(\omega_{0}\right), \Lambda^{p}\left(\omega_{1}\right)\right)_{f, p}=\Lambda^{p}\left(\omega_{0} f\left(\omega_{1} / \omega_{0}\right)\right)
$$

Finally, recall the definition of the Lorentz-Sharpley space associated to a symmetric Banach space $X$ whose fundamental function is the function parameter $\Phi_{X}(t)=\left\|\chi_{(0, t)}\right\|_{X}$ (cf. [20]):

$$
\Lambda^{p}(X)=\left\{f \in L_{0} ; f^{*} \in L_{p}^{*}\left(\Phi_{X}\right)\right\}=L_{p}^{*}\left(\Phi_{X}\right)^{s}
$$

and, in the case $p=\infty$,

$$
M(X)=\Lambda^{\infty}(X)=\left\{f \in L_{0} ; \sup _{s>0} f^{*}(s) \Phi_{X}(s)<\infty\right\} .
$$

By interpolation of couples of such spaces we obtain Lorentz spaces:

Theorem 22. Let $\left(X_{0}, X_{1}\right)$ be a couple of symmetric Banach spaces, $f$ a function parameter and $0<q \leq \infty$. Then $\left(M\left(X_{0}\right)^{d}, M\left(X_{1}\right)^{d}\right)$ is a Marcinkiewicz pair and

$$
\left(M\left(X_{0}\right), M\left(X_{1}\right)\right)_{f, q}=\left(L_{\infty}\left(\Phi_{X_{0}}\right), L_{\infty}\left(\Phi_{X_{1}}\right)\right)_{f, q}^{s} .
$$

If, additionally, the lower fundamental indices (defined as in [3]) satisfy $\alpha_{x_{1}}>0$ and $1 \leq p_{0}, p_{1} \leq \infty$, then

$$
\left(\Lambda^{p_{0}}\left(X_{0}\right), \Lambda^{p_{1}}\left(X_{1}\right)\right)_{f, q}=\left(L_{p_{0}}^{*}\left(\Phi_{X_{0}}\right), L_{p_{1}}^{*}\left(\Phi_{X_{1}}\right)\right)_{f, q}^{s} .
$$

Proof. The fundamental functions $\Phi_{x_{j}}$ are increasing and satisfy the $\Delta_{2}$-condition. It follows from Theorem 11 that $\left(M\left(X_{0}\right), M\left(X_{1}\right)\right)_{j, q}=\left(L_{\infty}\left(\Phi_{X_{0}}\right), L_{\infty}\left(\Phi_{X_{1}}\right)\right)_{j, q}^{s}$.

Now we assume that $X_{j}$ is a symmetric space such that $\alpha_{X_{j}}>0$. In this case, $\Phi_{X_{j}}$ is an increasing function such that 


$$
\inf _{x>0} \frac{\Phi_{X_{j}}(2 x)}{\Phi_{X_{j}}(x)}>1,
$$

it follows from Theorem 13 that $\left(L_{p_{0}}^{*}\left(\Phi_{X_{0}}\right)^{d}, L_{p_{1}}^{*}\left(\Phi_{X_{1}}\right)^{d}\right)$ is a Marcinkiewicz pair.

Final remark. For simplicity, in the hypotheses we have considered $\Delta_{2}$-weights, but we only apply $D_{2}$ as a bounded operator on the decreasing functions $f_{u}^{*}$ and in fact what is needed is only that the weights

$$
W(x)=\int_{0}^{x} \omega(t) d t
$$

satisfy this $\Delta_{2}$-condition. This fact follows from the identity

$$
\int_{0}^{\infty} f_{u}^{*}(t)^{p} \omega(t) d t=p \int_{0}^{\infty} y^{p-1}\left(\int_{0}^{\lambda_{j}^{*}(y)} \omega(t) d t\right) d y
$$

with $\lambda_{f}^{u}(y)=\int_{[x ;|f(x)|>y]} u(x) d x($ cf. [7, Theorem 2.1]).

\section{REFERENCES}

1. K. ANDERSEN, Weighted generalized Hardy inequalities for nonincreasing functions, Canad. J. Math. 43 (1991), 1121-1135.

2. M. Ariño and B. MUCKenhoupt, Maximal functions on classical Lorentz spaces and Hardy's inequality for nonincreasing functions, Trans. Amer. Math. Soc. 320 (1990), 727-735.

3. C. BenNeTt and R. Sharpley, Interpolation of Operators (Academic Press, 1988).

4. J. BERGH and J. LöFSTRÖM, Interpolation spaces. An introduction (Springer Verlag, 1976).

5. YU. A. BRUDNY and N. YA. KRUGLJAK, Interpolation Functors and Interpolation Spaces (North-Holland, 1991).

6. M. J. Carro and J. Soria, Boundedness of some integral operators, Canad. J. Math. 45 (1993), 1155-1166.

7. M. J. Carro and J. Soria, Weighted Lorentz Spaces and the Hardy Operator, J. Funct. Anal. 112 (1993), 480-494.

8. J. CERDÀ and J. MARTín, Interpolation of operators on decreasing functions, Math. Scand. 78 (1996), 233-245.

9. J. Cerdì and J. Martín, Conjugate Hardy's inequalities with decreasing weights, Proc. Amer. Math. Soc. 126 (1998), 2341-2344.

10. D. Freitag, Real Interpolation of weighted $L_{p}^{*}$-spaces, Math. Nachr. 86 (1978), 15-18.

11. A. J. García Del Amo, On reverse Hardy's inequality, Collect. Math. 44 (1993), 115-123.

12. J. Gustavson, A function parameter in connection with interpolation of Banach spaces, Math. Scand. 42 (1978), 289-305. 
13. H. Hudzik and L. Maligranda, An interpolation theorem in symmetric function $F$-spaces, Proc. Amer. Math. Soc. 110 (1990), 89-96.

14. S. G. Krein, Ju. I. Petunin and E. M. Semenov, Interpolation of Linear Operators (Transl. Math. Monogr. 54, Amer. Math. Soc., 1982).

15. L. Maligranda and L. E. Persson, Real interpolation between weighted $L^{p}$ and Lorentz spaces, Bull. Pol. Acad. Sci. 35 (1987), 765-778.

16. C. MERUCCI, Interpolation réelle avec fonction paramètre: réitération et applications aux espaces $\Lambda^{p}(\Phi)(0<p \leq \infty)$, C. R. Acad. Sci. Paris 295 (1982), 427-430.

17. C. J. Neugebauer, Some classical operators on Lorentz space, Forum Math. 4 (1992), $135-146$.

18. Y. SAGHER, An application of interpolation theory to Fourier series, Studia Math. 38 (1972), 169-181.

19. E. SAWyer, Boundedness of classical operators on classical Lorentz spaces, Studia Math. 96 (1990), 145-158.

20. R. Sharpley, Spaces $\Lambda_{2}(X)$ and interpolation, J. Funct. Anal. 11 (1972), 479-513.

21. V. Stepanov, Integral operators on the cone of monotone functions, J. London Math. Soc. 48 (1993), 465-487.

Departament de Matemàtica Aplicada i Anàlisi

UNIVERSTTAT DE BARCELONA

E-08071 BARCELONA

SPAIN

E-mail addresses: cerda@cerber.mat.ub.es, jmartin@cerber.mat.ub.es 